\title{
Therapeutic effects of adipose-derived stem cells pretreated with pioglitazone in an emphysema mouse model
}

\author{
Yoonki Hong ${ }^{1,4}$, You-Sun Kim ${ }^{2,4}$, Seok-Ho Hong ${ }^{1}$ and Yeon-Mok $\mathrm{Oh}^{3}$
}

There is no therapy currently available that influences the natural history of disease progression in patients with chronic obstructive pulmonary disease (COPD). Although stem cell therapy is considered a potential therapeutic option in COPD, there are no clinical trials proving definitive therapeutic effects in patients with COPD. Recently, it was reported that pioglitazone might potentiate the therapeutic effects of stem cells in patients with heart or liver disease. To test the capacity of pioglitazone pretreatment of stem cells for emphysema repair, we evaluated the therapeutic effects of pioglitazone-pretreated human adiposederived mesenchymal stem cells (ASCs) on elastase-induced or cigarette smoke-induced emphysema in mice. We also investigated the mechanisms of action of pioglitazone-pretreated ASCs. Pioglitazone-pretreated ASCs had a more potent therapeutic effect than non-pretreated ASCs in the repair of both elastase-induced and smoke-induced emphysema models (mean linear intercept, $78.1 \pm 2.5 \mu \mathrm{m}$ vs $83.2 \pm 2.6 \mu \mathrm{m}$ in elastase models and $75.6 \pm 1.4 \mu \mathrm{m}$ vs $80.5 \pm 3.2 \mu \mathrm{m}$ in smoke models, $P<0.05$ ). Furthermore, we showed that pioglitazone-pretreated ASCs increased vascular endothelial growth factor (VEGF) production both in vitro and in mouse lungs in the smoke-induced emphysema model. Pioglitazone-pretreated ASCs may have more potent therapeutic effects than non-pretreated ASCs in emphysema mouse models.

Experimental \& Molecular Medicine (2016) 48, e266; doi:10.1038/emm.2016.93; published online 21 October 2016

\section{INTRODUCTION}

Chronic obstructive pulmonary disease (COPD) is a disease state characterized by airflow limitation that is not fully reversible. ${ }^{1}$ COPD is a major cause of morbidity and mortality worldwide, and its incidence is estimated to rise to the third leading cause of death worldwide by $2030 .^{2}$ Although the pathogenesis of COPD is incompletely understood, emphysema and small-airway disease are known to be the main pathological processes for developing airflow limitations. ${ }^{3}$ The mechanisms involved in emphysema development are persistent inflammation, extracellular matrix proteolysis, apoptosis and ineffective repair. ${ }^{4,5}$ Although COPD is an important public health problem, there is no curative therapy for patients with COPD, and current therapeutic approaches are mostly focused on improving symptoms and decreasing the frequency of exacerbations. ${ }^{1}$

Recent advances in stem cell therapy may lead to a promising therapeutic option for more broad and definitive treatments of COPD considering that stem cells can differentiate into multiple lineages ${ }^{6}$ and repair injured tissue via paracrine actions. ${ }^{7}$ The therapeutic effects of stem-cell-based cell therapy have been shown in various organ diseases, such as diseases of the heart, bone, liver and lung. ${ }^{8-12}$ Mesenchymal stem cells (MSCs) from bone marrow and adipose tissue have also demonstrated a therapeutic effect for COPD in an experimental cigarette smoke-induced model and elastaseinduced emphysema model. ${ }^{13-17}$ Our previous research also demonstrated that bone-marrow-derived MSCs repaired cigarette smoke-induced emphysema. ${ }^{18}$ To date, clinical trials for stem cells in the area of COPD have not demonstrated definitive therapeutic effects, such as pulmonary function improvement or mortality reduction, although systemic administration of MSCs was found to be safe in patients with COPD. ${ }^{19}$ However, clinical trials are ongoing.

It is suggested that the therapeutic impact of MSCs is mediated by their ability to differentiate into tissues and,

\footnotetext{
${ }^{1}$ Department of Internal Medicine, Kangwon National University Hospital, Chuncheon, Korea; ${ }^{2}$ Asan Institute for Life Sciences, Department of Pulmonary and Critical Care Medicine, University of Ulsan College of Medicine, Seoul, Korea and ${ }^{3}$ Department of Pulmonary and Critical Care Medicine, Asan Medical Center, Seoul, Korea

${ }^{4}$ These authors contributed equally to this work.

Correspondence: Professor Y-M Oh, Department of Pulmonary and Critical Care Medicine, Asan Medical Center, 86 Asanbyeongwon-gil, Songpa-gu, Seoul 138-736, South Korea.

E-mail: ymoh55@amc.seoul.kr

Received 31 March 2016; revised 17 May 2016; accepted 19 May 2016
} 
potentially, by their trophic, paracrine and anti-apoptotic functions. ${ }^{20}$ More advanced methods of manufacturing MSCs, including increasing differentiation efficiency, more persistent engraftment and/or augmentation of the paracrine effect, may be required to increase the therapeutic effects of MSCs for application in clinical practice. We have previously reported that paracrine effects might be more important than stem cell engraftment, ${ }^{18}$ the distribution of intravenously injected MSCs, ${ }^{21}$ and the optimal dose of MSCs in elastase-induced emphysema models; ${ }^{22}$ these data may be helpful for further research on MSCs for patients with COPD. However, much remains to be determined for the clinical application of MSCs in patients with COPD.

Because the preferred culture methods to develop the maximal paracrine effects of human MSCs are still undetermined, ${ }^{20}$ clinical trials may show a substantial paracrine effect of MSCs if an adequate method to potentiate stem cells is identified. Recently, it has been suggested that peroxisome proliferator-activated receptor- $\gamma$ (PPAR- $\gamma$ ) activation changed the phenotype of mesenchymal cells during cell differentiation. Furthermore, it was reported that transplantation of pioglitazone-pretreated MSCs significantly improved cardiac function and may be a promising cardiac stem cell source to promote cardiomyogenesis or augmentation of paracrine functions. ${ }^{23}$

Therefore, we aimed to identify the capacities of pioglitazone pretreatment of stem cells in COPD. In this study, we intravenously injected with adipose-derived stem cells (ASCs) pretreated with pioglitazone into mice with elastase-induced or smoke-induced emphysema, identified the therapeutic effects and evaluated the mechanisms of action of pioglitazonepretreated ASCs (pioASCs).

\section{MATERIALS AND METHODS}

\section{Cell culture and pretreatment with pioglitazone}

ASCs were purchased from Invitrogen (Carlsbad, CA, USA) and cultured using MesenPRO RS Medium with supplied supplements (Invitrogen). ASCs were fed every 3-4 days with fresh media and were subcultured with $0.05 \%$ trypsin-EDTA (Gibco Life Technologies, Grand Island, NY, USA). For pioASCs, the ASC culture medium was treated with $3 \mu \mathrm{moll}^{-1}$ pioglitazone for 1 week. MLE-12 airwayepithelial cells were purchased from ATCC (Manassas, VA, USA), cultured with the recommended complete growth medium and subcultured with $0.05 \%$ Trypsin-EDTA (Gibco Life Technologies).

\section{Preparation of conditioned media}

At $70 \%$ confluence, ASCs were washed three times with phosphatebuffered saline and incubated with MesenPRO RS Medium. After $24 \mathrm{~h}$, ASC-conditioned media (ASC-CM) were collected, filtered through a $0.22-\mu \mathrm{m}$ filter, and concentrated aseptically using an Amicon Ultracentrifugal filter device with a molecular weight cutoff of $10 \mathrm{kDa}$ (Amicon, Beverly, MA, USA).

\section{Proliferation assay}

We performed a CCK- 8 assay to measure proliferation rates. The MLE-12 cell line was incubated for $24 \mathrm{~h}$ with ASC-CM or pioASCCM; then, CCK-8 was mixed with medium (1:10, CCK-8:medium) and incubated for $2 \mathrm{~h}$. Absorbance at $450 \mathrm{~nm}$ was measured using a microplate reader (PerkinElmer, Waltham, MA, USA).

\section{Mouse model}

The Institutional Animal Care and Use Committee of Asan Medical Center approved all mouse experiments in this study. C57BL/6 mice were purchased from Orient Bio (Seongnam, Korea). Mice were housed in a specific pathogen-free facility. For the elastase-induced mouse model, as described previously, ${ }^{21}$ 6-week-old female C57BL/6 J mice were intratracheally injected with $0.4 \mathrm{U}$ of porcine pancreatic elastase (Sigma-Aldrich, St Louis, MO, USA) at day 0, followed by an intravenous injection of $1 \times 10^{5}$ ASCs at day 7 , and then killed at day 14 for lung harvest. For the smoke-induced emphysema model, as described previously, ${ }^{18}$ the mice were exposed to cigarette smoke 5 days per week for 6 months from commercially purchased cigarettes that contained $8.5 \mathrm{mg}$ of tar and $0.9 \mathrm{mg}$ of nicotine (Eighty Eight Lights, KT\&G, Daejeon, Korea). After exposure to cigarette smoke for 6 months, the mice were intravenously injected with $1 \times 10^{5}$ ASCs and then killed on day 7 .

\section{Histology and quantification of emphysema}

Lung samples for histology were obtained using a previously described method. ${ }^{21}$ In brief, the perfused lungs were inflated with $0.5 \%$ lowmelting agarose, fixed with $4 \%$ formalin and embedded in paraffin. Lung sections of 6- $\mu \mathrm{m}$ thickness were stained with hematoxylin and eosin. Mean linear intercepts (MLIs) were determined using microscopy images.

\section{HGF, FGF2 and VEGF measurement}

Ten micrograms of protein from lung tissue were examined using enzyme-linked immunosorbent assay (ELISA). The hepatocyte growth factor (HGF), fibroblast growth factor 2 (FGF2) and vascular endothelial growth factor (VEGF) levels in the lung protein lysates were measured using ELISA according to the manufacturer's instructions (R\&D Systems, Minneapolis, MN, USA).

\section{Caspase-3/7 activity measurement}

Caspase-3/7 measurement has been previously described. ${ }^{21}$ Ten micrograms of protein, prepared with a cell lysis buffer (Cell Signaling Technology, Danvers, MA, USA) and quantified based on the Bradford assay, were incubated with caspase-3/7 substrate diluted with caspase-3/7 buffer in black multiwell plates (Promega, Madison, WI, USA). After $5 \mathrm{~h}$, the fluorescence of each sample was measured using a fluorometer.

\section{Data analysis}

Statistical analyses were performed using GraphPad Prism ver. 5 software (GraphPad Software Inc., La Jolla, CA, USA). The data are presented as the mean \pm standard error. The Mann-Whitney $U$ test was used to compare both groups, and statistical significance was set at $P<0.05$.

\section{RESULTS}

\section{Lung epithelial cell proliferation in vitro}

To identify the effects of pioglitazone pretreatment on ASCs in vitro, the murine lung epithelial MLE-12 cell line was cultured in ASC-CM and pioASC-CM. Proliferation of lung epithelial cells was higher in the group treated with pioASC-CM than in the group treated with ASC-CM $(P=0.028$; Figure 1a and $b)$. 
a
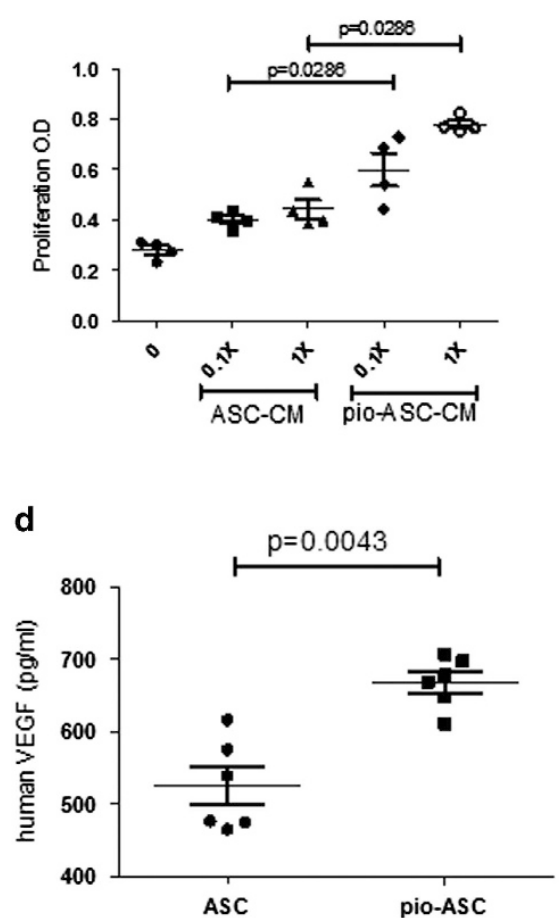

b

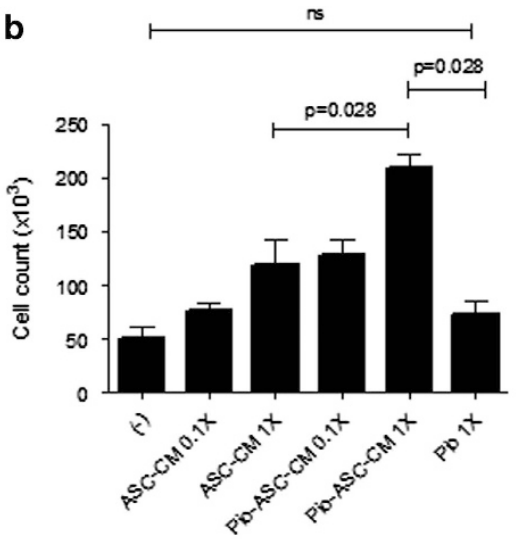

e

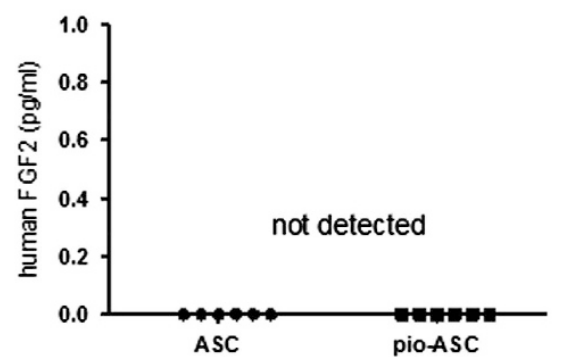

C

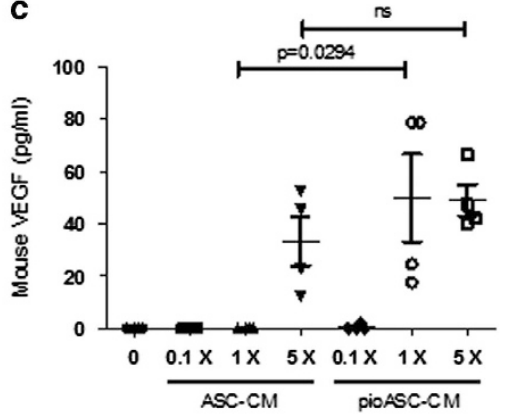

Figure 1 The effects of pioglitazone pretreatment on human adipose-derived stem cell (ASCs) in vitro. The murine lung epithelial MLE-12 cell line was cultured in conditioned media with ASCS (ASC-CM) or pioglitazone-pretreated CM with ASCs (pioASC-CM). Proliferation of lung epithelial cells was increased in the pioASC-CM compared with the ASC-CM. $(P=0.028)$ (a and b). Mouse vascular endothelial growth factor (VEGF) production in pioASC-CM $(\times 1)$ was significantly higher than in ASC-CM, $(P=0.029)(\mathbf{c})$, and the human VEGF level in lung epithelial cells was higher in pioASC-CM than in ASC-CM. $(P=0.004)$ (d) And, human fibroblast growth factor 2 (FGF2) production was not detected in either group (e).

Mouse VEGF levels from lung epithelial cells $(\times 1)$ were higher in mice treated with pioASC-CM than in mice treated with ASC-CM $(P=0.029)$ (Figure 1c). Production of human VEGF was significantly higher in mice treated with pioASCs than ASCs $(P=0.004)$ (Figure 1d). However, human FGF2 production was not detected in either group (Figure 1e).

Repair of elastase-induced emphysema by pioASCs

In the elastase-induced mouse emphysema model, mice treated with ASCs and pioASCs showed improved alveolar regeneration compared with elastase-only mice (Figure 2a). Alveolar destruction was measured quantitatively by MLI. The elastaseonly mice showed markedly increased MLI $(90.8 \pm 3.3)$ compared with the MLI $(58.4 \pm 0.7 \mu \mathrm{m})$ of control mice, and the MLI levels were decreased $(83.2 \pm 2.6 \mu \mathrm{m})$ in the mice treated with ASCs and further decreased $(78.1 \pm 2.5 \mu \mathrm{m})$ in the mice treated with pioASCs $(P<0.05)$ (Figure $2 \mathrm{~b})$.

\section{The mechanisms of pioASCs in the elastase-induced} emphysema model

To identify the mechanisms of action of pioASCs in the mice with elastase-induced emphysema, proteins and messenger RNA were obtained from lung tissue of the four groups (control group, elastase-only group, ASC-treated elastase group and pioASC-treated elastase group). The mechanisms of action on regeneration were evaluated by assessing growth factors and apoptosis. First, production of the growth factors HGF, FGF2 and VEGF were measured in lung homogenate using ELISA. HGF and FGF2 levels were not significantly different among the four groups (Figure $3 b$ and $c$ ). The VEGF level in lung tissue was significantly decreased in the elastase-only group compared with the control group, and it was increased in the pioASC-treated and ASC-treated mouse groups $(P<0.05$; Figure 3a). There was no significant difference between the pioASC-treated and ASC-treated mouse groups. Second, caspase-3/7-dependent apoptosis was evaluated. Caspase-3/7 activity was significantly increased in the elastase-only group compared with the control group, and it was significantly decreased in the pioASC-treated and ASC-treated groups compared with the elastase-only group $(P<0.05)$. No significant difference was seen between the elastase-only group and the pioASC-treated group (Figure 3d).

\section{Effect of pioASCs on smoke-induced emphysema}

For the smoke-induced emphysema mouse model, similar to the elastase model, pioASC-treated mice showed improved alveolar regeneration compared with ASC-treated mice (Figure 4a). In addition, pioASC-treated mice had significantly 

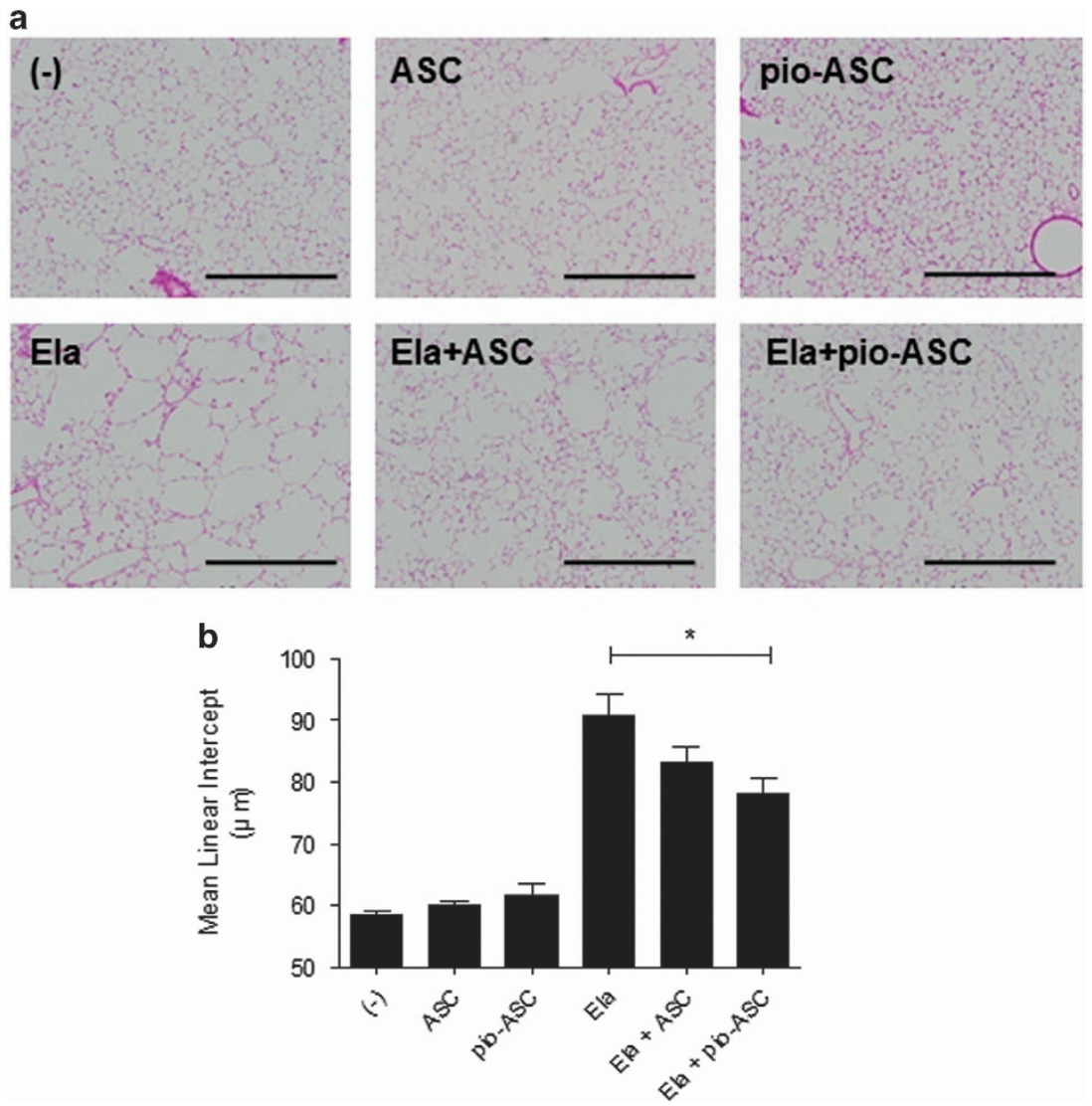

Figure 2 The therapeutic effects of pioglitazone pretreatment on human adipose-derived stem cells (ASCs) in mice with elastase-induced emphysema. C57BL/6 J mice were intratracheally injected with $0.4 \mathrm{U}$ of elastase on day 0 and then intravenously injected with ASCs or pioglitazone-pretreated ASCs (pioASCs) on day 7. Data are measures of the histological staining with hematoxylin and eosin in lung sections on day 14. (a) Control $(-, n=4)$, Ela (elastase only, $n=9$ ), ASC (ASC only, $n=7$ ), pioASC (pioASC only, $n=10$ ), Ela+ASC (elastase+ASC, $n=8$ ) and Ela+pioASC (elastase+pioASC, $n=9$ ). Scale bars, $0.5 \mathrm{~mm}$. (b) Morphometic analysis of the mean linear intercept (MLIs). Values are presented as the mean \pm s.e.m. ${ }^{*} P<0.05$ was statistically significant in the comparison between groups.

lower MLI $(75.6 \pm 1.4)$ than the MLI $(80.5 \pm 3.2)$ of ASCtreated mice $(P<0.05$; Figure $4 \mathrm{~b})$. In the smoke-induced emphysema model, HGF, FGF2 and VEGF production related to lung regeneration were also measured in lung homogenate using ELISA. HGF, FGF2 and VEGF levels in lung tissue were significantly increased in the pioASC-treated mice compared with the elastase-only mice and the ASC-treated mice (Figure 4c-e).

\section{DISCUSSION}

In this study, we showed that pioASCs have more potent therapeutic effects than ASCs for repair of alveolar destruction in emphysema mouse models. In addition, we showed that these effects may be mediated via restored imbalance of protease/antiprotease, decreased apoptotic activity and increased production of growth factors, suggesting augmentation of paracrine effects by pioASCs. Furthermore, we suggest that VEGF may play an important role in potentiating pioASCs because increased VEGF production was shown in pioASC-CM compared with ASC-CM in vitro, and mice treated with pioASCs had increased VEGF production compared with the mice treated with ASCs in the smoke-induced emphysema model. We showed, for the first time, that pioASCs were more effective in alleviating emphysema compared with ASCs. These data may be of use in expanding the efficacy of ASCs in clinical studies of COPD.

In COPD, stem cell therapy is an interesting alternative therapy because there is no curative therapy in current treatments of COPD, and stem cell therapy may also be a candidate therapy because of its capacity for repairing injured tissue. The therapeutic effects of MSCs derived from bone marrow and adipose tissue have been shown in mouse models of emphysema. ${ }^{13-18}$ However, because there has been no demonstrated evidence of clinical benefit in patients with COPD, we attempted to elucidate the mechanisms of action and/or find more effective methods of manufacturing stem cells. The reported mechanisms of action of stem cells in COPD studies are inconsistent. However, many studies have focused on the paracrine effects of MSCs versus effects by differentiation or engraftments when explaining stem cell mechanisms of action in COPD models; these studies reported increased levels of growth factors, ${ }^{16}$ similar therapeutic effects of cell-free MSC-CM ${ }^{18}$ and identification of limited engraftment rates of MSCs. ${ }^{21}$ Because MSCs produce many 


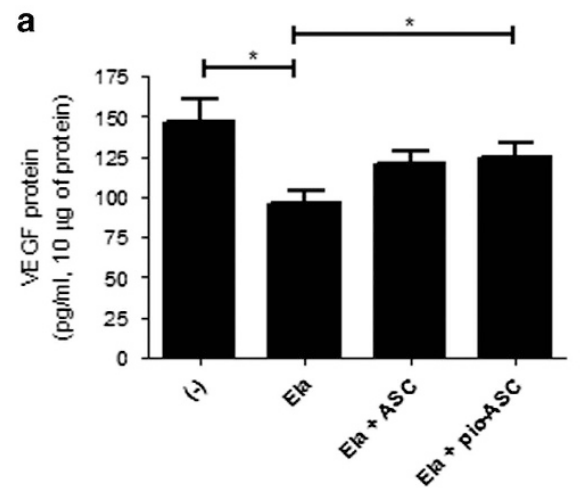

C

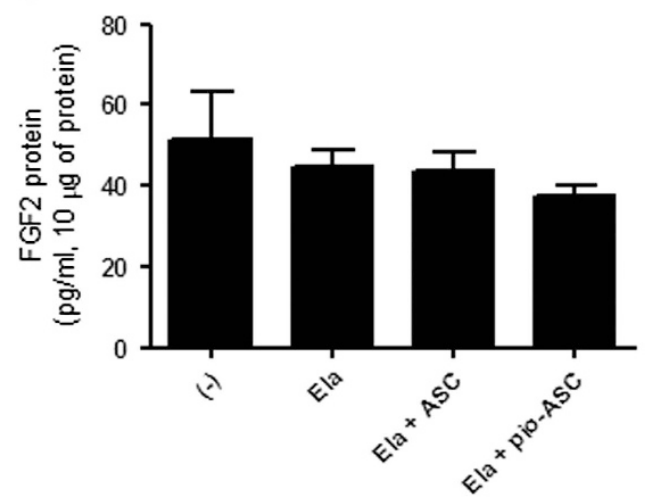

b

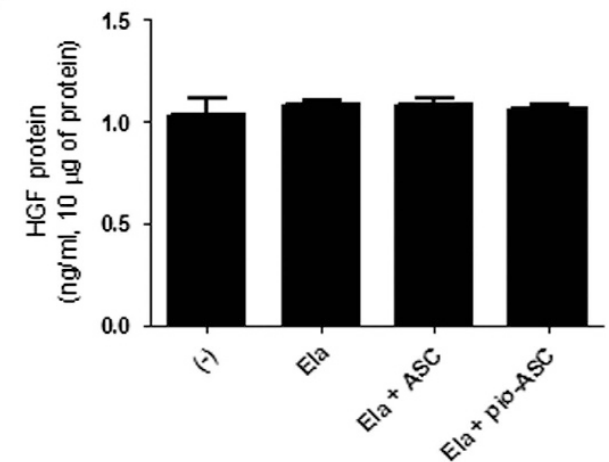

d

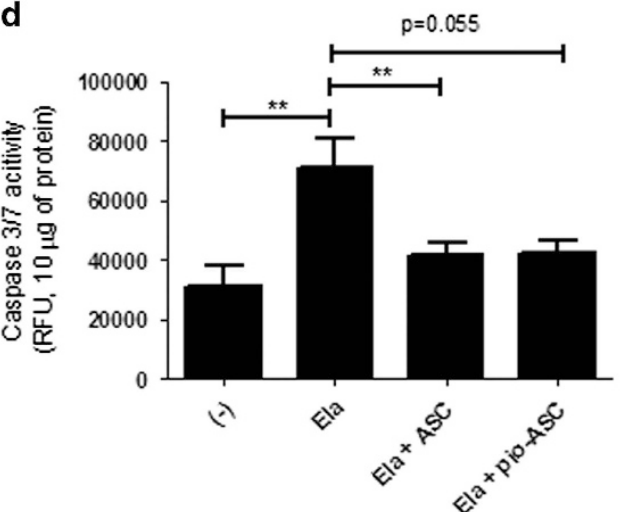

Figure 3 The production of growth factors in elastase-induced emphysema with adipose-derived stem cells (ASCs) or pioglitazonepretreated ASCs (pioASCs). C57BL/6 J mice were intratracheally injected with $0.4 \mathrm{U}$ of elastase on day 0 and then intravenously injected with ASC or pioASCs on day 7. Lung tissue was collected on day 14 ( $n=5-9$ per group). The growth factors were measured with enzymelinked immunosorbent assay (ELISA) for vascular endothelial growth factor (VEGF) (a), hepatocyte growth factor (HGF) (b) and fibroblast growth factor 2 (FGF2) (c). Caspase-3/7 activity was measured with fluorimetric enzymatic assay and normalized by protein concentration in lung homogenates. (d) Values are presented as the mean \pm s.e.m. * and ** denote statistical significance $(P<0.05)$ for the comparison between groups. ( - ): control; Ela, elastase only; Ela+ASC, elastase+ASC; Ela+pioASC, elastase+pioASC.

inflammatory cytokines and growth factors, ${ }^{20,24}$ it is understood that MSCs assist and modulate the regenerative environment via paracrine mechanisms. ${ }^{20}$ Among growth factors HGF, FGF2 and VEGF are known to be critical growth factors to repair lung destruction in emphysema models. ${ }^{25-27}$ In particular, VEGF is known to be an important factor in airway and pulmonary vasculature development. ${ }^{25}$ Furthermore, according to previous reports that inhibition of VEGF receptors induced emphysema features, ${ }^{28}$ and ASC delivery was protective of damage in the VEGF inhibition model of emphysema, ${ }^{17}$ VEGF induced by stem cells is crucial for protection of lung damage. Our previous research also showed that the therapeutic effects of MSCs were related to an increase in VEGF production. In addition, our current study emphasizes the role of VEGF in therapeutic effects of ASCs and in potentiating the effects of pioASCs, showing greater levels of VEGF production in pioASC-CM in vitro and in the smoke-induced mouse model. Our current study, however, did not show differences in the levels of VEGF production between ASC- and pioASC-treated groups in the elastase-induced model, although the level of VEGF production was increased in the ASC- and pioASCtreated groups. VEGF signaling is mediated by its receptors, such as VEGF receptor-1, -2 and -3 . Therefore, there may be differences in the effects of VEGF following expression of each VEGF receptor. However, we did not confirm VEGF receptor expression in lungs from elastase- and smoke-induced models. It remains to be studied further.

Pioglitazone acts as an agonist of PPAR- $\gamma$ and is known to enhance adipocyte differentiation. ${ }^{29}$ Because PPAR- $\gamma$ is also known to be expressed in MSCs, ${ }^{30}$ it has been suggested that PPAR- $\gamma$ activation, using pioglitazone, changes the phenotype of mesenchymal cells during cell differentiation. Several studies have reported that pioglitazone enhances transdifferentiation of stem cells and improves therapeutic functions. Shinmura et $a .^{23}$ reported that transplantation of pioglitazonepretreated bone-marrow MSCs repaired heart tissue in an myocardial infarction model in vivo. Werner et al. ${ }^{31}$ reported that pioglitazone increased the number and function of bonemarrow-derived endothelial progenitor cells in patients with coronary artery disease and that this effect represents a potential regenerative mechanism in atherosclerosis. Recently, Vosough et al. ${ }^{32}$ also reported that transplantation of MSCs with pioglitazone in cirrhotic patients was safe and feasible. These clinical studies showed that stem cell therapy combined 
a
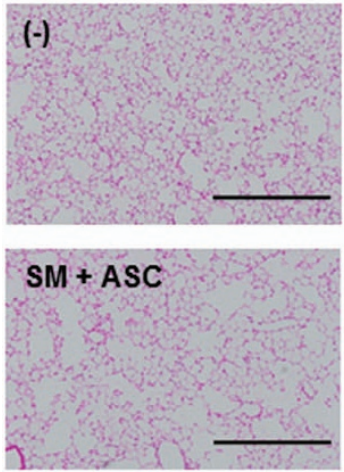

SM

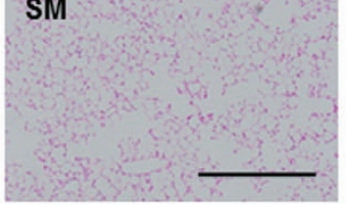

SM + pio-ASC

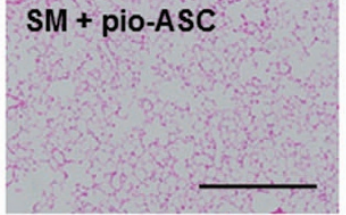

b

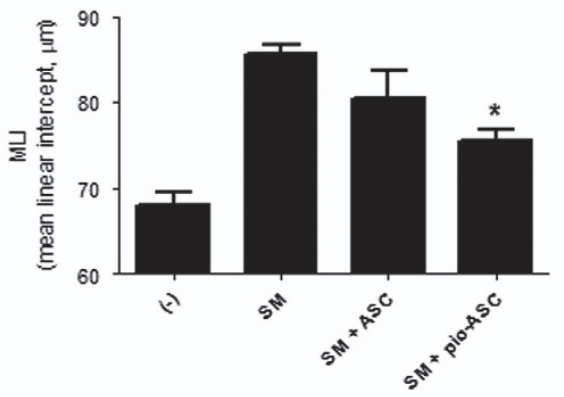

c

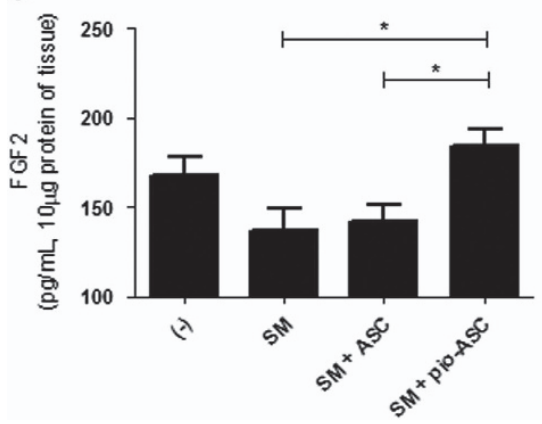

d

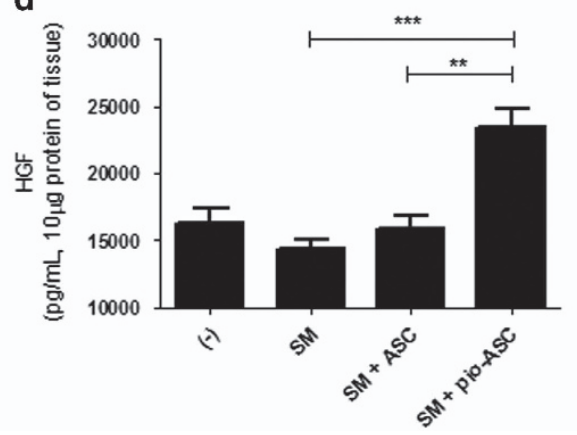

e

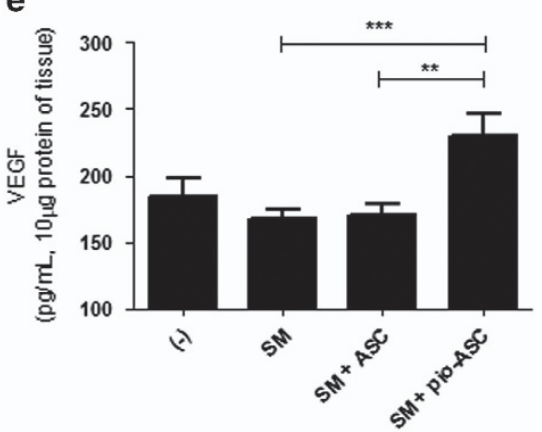

Figure 4 The therapeutic effects and mechanisms of action of human adipose-derived stem cells (ASCs) or pioglitazone-pretreated ASCs (pioASCs) in mice with smoke-induced emphysema. C57BL/6 J mice were exposed to cigarette smoke for 6 months and were then treated with ASCs or pioASCs. Shown here is the histological assessment at 2 months of lung sections stained with hematoxylin and eosin. (a) Control $(-, n=5)$, SM (smoking only, $n=4)$ ), SM+ASC (smoking+ASC, $n=4$ ) and SM+pioASC (smoking+pioASC, $n=5$ ). Scale bars, $0.5 \mathrm{~mm}$. (b) Morphometic analysis of the mean linear intercept (MLIs). Values are presented as the mean \pm s.e.m. ${ }^{*} P<0.05$ was statistically significant in the comparison between groups. The growth factors were measured with enzyme-linked immunosorbent assay (ELISA) for vascular endothelial growth factor (VEGF) (c), hepatocyte growth factor (HGF) (d) and fibroblast growth factor 2 (FGF2) (e). Values are presented as the mean \pm s.e.m. ${ }^{*},{ }^{* *}$ and ${ }^{* * *}$ denote statistical significance $(P<0.05)$ for the comparison between groups. $(-)$ : control; SM, smoke only; SM+ASC, smoke+ASC; SM+pioASC, smoke+pioASC.

with pioglitazone might potentiate the therapeutic effects of stem cells in heart or liver disease. Moreover, Shinmura et al. ${ }^{23}$ reported that pioglitazone increased the cardiomyogenic transdifferentiation of human bone-marrow MSCs via PPAR- $\gamma$ activation in vitro, and Wang et al. ${ }^{33}$ also reported that pioglitazone stimulated the transdifferentiation of bonemarrow MSCs into adipocytes in a rat model. These studies suggest that pioglitazone facilitates MSCs to core cells of injured tissue and improves organ function through differentiation. However, the precise mechanisms of action of pioglitazone on stem cells remain to be elucidated. Recently, Hou et $a .^{34}$ reported that activation of PPAR- $\gamma$ enhanced survival and therapeutic efficacy of MSCs via upregulation of Cx43 expression. Another possible explanation is that that the beneficial effects of pioglitazone on stem cell therapy outcomes is that pioglitazone augments the paracrine effect of MSCs. In our current research, we showed that pretreatment with pioglitazone in stem cell culture media increased therapeutic effects in damaged lung tissue and increased production of growth factors. These findings suggest that the beneficial effects of pioglitazone on lung tissue may be because of augmentation of paracrine effects of ASCs. ASC potentiation by means of pioglitazone may lead us to expand the efficacy of ASCs in clinical studies.

In conclusion, we report that pioASCs have more potent therapeutic effects than ASCs in emphysema mouse models. Furthermore, we suggest that the paracrine effects of ASCs, particularly on the production of growth factors such as VEGF, may have an important role in the mechanism that potentiates ASCs by pioglitazone. These data may be helpful in expanding the efficacy of ASCs in a clinical study of COPD.

\section{CONFLICT OF INTEREST}

The authors declare no conflict of interest.

\section{ACKNOWLEDGEMENTS}

We thank the members of the Asan Medical Center animal facility for their technical expertise. This study was supported by a grant from the Korean Health Technology R\&D Project, Ministry of Health \& Welfare and Republic of Korea (no. HI12C0169). 
1 Rabe KF, Hurd S, Anzueto A, Barnes PJ, Buist SA, Calverley P et al. Global strategy for the diagnosis, management, and prevention of chronic obstructive pulmonary disease: GOLD executive summary. Am J Respir Crit Care Med 2007; 176: 532-555.

2 Mannino DM, Buist AS. Global burden of COPD: risk factors, prevalence, and future trends. Lancet 2007; 370: 765-773.

3 Hogg JC. Pathophysiology of airflow limitation in chronic obstructive pulmonary disease. Lancet 2004; 364: 709-721.

4 MacNee W, Tuder RM. New paradigms in the pathogenesis of chronic obstructive pulmonary disease. Proc Am Thorac Soc 2009; 6: 527-531.

5 Yoshida T, Tuder RM. Pathobiology of cigarette smoke-induced chronic obstructive pulmonary disease. Physiol Rev 2007; 87: 1047-1082.

6 Anjos-Afonso F, Siapati EK, Bonnet D. In vivo contribution of murine mesenchymal stem cells into multiple cell-types under minimal damage conditions. J Cell Sci 2004; 117: 5655-5664.

7 Gnecchi M, Zhang Z, Ni A, Dzau VJ. Paracrine mechanisms in adult stem cell signaling and therapy. Circ Res 2008; 103: 1204-1219.

8 Kocher AA, Schuster MD, Szabolcs MJ, Takuma S, Burkhoff D, Wang J et al. Neovascularization of ischemic myocardium by human bone-marrow-derived angioblasts prevents cardiomyocyte apoptosis, reduces remodeling and improves cardiac function. Nat Med 2001; 7: 430-436.

9 Orlic D, Kajstura J, Chimenti S, Limana F, Jakoniuk I, Quaini F et al. Mobilized bone marrow cells repair the infarcted heart, improving function and survival. Proc Natl Acad Sci USA 2001; 98: 10344-10349.

10 Horwitz EM, Prockop DJ, Fitzpatrick LA, Koo WW, Gordon PL, Neel M et al. Transplantability and therapeutic effects of bone marrow-derived mesenchymal cells in children with osteogenesis imperfecta. Nat Med 1999; 5: 309-313.

11 Zhao DC, Lei JX, Chen R, Yu WH, Zhang XM, Li SN et al. Bone marrowderived mesenchymal stem cells protect against experimental liver fibrosis in rats. World J Gastroenterol 2005; 11: 3431-3440.

12 Matthay MA, Goolaerts A, Howard JP, Lee JW. Mesenchymal stem cells for acute lung injury: preclinical evidence. Crit Care Med 2010; 38 : S569-S573.

13 Longhini-Dos-Santos N, Barbosa-de-Oliveira VA, Kozma RH, Faria CA, Stessuk T, Frei $\mathrm{F}$ et al. Cell therapy with bone marrow mononuclear cells in elastase-induced pulmonary emphysema. Stem Cell Rev 2013; 9: 210-218.

14 Cruz FF, Antunes MA, Abreu SC, Fujisaki LC, Silva JD, Xisto DG et al. Protective effects of bone marrow mononuclear cell therapy on lung and heart in an elastase-induced emphysema model. Respir Physiol Neurobiol 2012; 182: 26-36.

15 Shigemura N, Okumura M, Mizuno S, Imanishi Y, Nakamura T, Sawa Y. Autologous transplantation of adipose tissue-derived stromal cells ameliorates pulmonary emphysema. Am J Transplant 2006; 6: 2592-2600.

16 Katsha AM, Ohkouchi S, Xin H, Kanehira M, Sun R, Nukiwa T et al. Paracrine factors of multipotent stromal cells ameliorate lung injury in an elastase-induced emphysema model. Mol Ther 2011; 19: 196-203.

17 Schweitzer KS, Johnstone BH, Garrison J, Rush NI, Cooper S, Traktuev DO et al. Adipose stem cell treatment in mice attenuates lung and systemic injury induced by cigarette smoking. Am J Respir Crit Care Med 2011; 183: 215-225.

18 Huh JW, Kim SY, Lee JH, Lee JS, Van Ta Q, Kim M et al. Bone marrow cells repair cigarette smoke-induced emphysema in rats. Am J Physiol Lung Cell Mol Physiol 2011; 301: L255-L266.

19 Weiss DJ, Casaburi R, Flannery R, LeRoux-Williams M, Tashkin DP. A placebo-controlled, randomized trial of mesenchymal stem cells in COPD. Chest 2013; 143: 1590-1598.

20 Murphy MB, Moncivais K, Caplan Al. Mesenchymal stem cells: environmentally responsive therapeutics for regenerative medicine. Exp Mol Med 2013; 45: e54.
21 Kim YS, Kim JY, Shin DM, Huh JW, Lee SW, Oh YM. Tracking intravenous adipose-derived mesenchymal stem cells in a model of elastase-induced emphysema. Tuberc Respir Dis (Seoul) 2014; 77: 116-123.

22 Kim YS, Kim JY, Huh JW, Lee SW, Choi SJ, Oh YM. The therapeutic effects of optimal dose of mesenchymal stem cells in a murine model of an elastase induced-emphysema. Tuberc Respir Dis (Seoul) 2015; 78 : 239-245.

23 Shinmura D, Togashi I, Miyoshi S, Nishiyama N, Hida N, Tsuji H et al. Pretreatment of human mesenchymal stem cells with pioglitazone improved efficiency of cardiomyogenic transdifferentiation and cardiac function. Stem Cells 2011; 29: 357-366.

24 Haynesworth SE, Baber MA, Caplan Al. Cytokine expression by human marrow-derived mesenchymal progenitor cells in vitro: effects of dexamethasone and IL-1 alpha. J Cell Physiol 1996; 166: 585-592.

25 Hegab AE, Kubo H, Yamaya M, Asada M, He M, Fujino N et al. Intranasal HGF administration ameliorates the physiologic and morphologic changes in lung emphysema. Mol Ther 2008; 16: 1417-1426.

26 Muyal JP, Muyal V, Kotnala S, Kumar D, Bhardwaj H. Therapeutic potential of growth factors in pulmonary emphysematous condition. Lung 2013; 191: 147-163.

27 Guan XJ, Song L, Han FF, Cui ZL, Chen X, Guo XJ et al. Mesenchymal stem cells protect cigarette smoke-damaged lung and pulmonary function partly via VEGF-VEGF receptors. J Cell Biochem 2013; 114: 323-335.

28 Kasahara Y, Tuder RM, Taraseviciene-Stewart L, Le Cras TD, Abman S, Hirth PK et al. Inhibition of VEGF receptors causes lung cell apoptosis and emphysema. J Clin Invest 2000; 106: 1311-1319.

29 Yamauchi T, Kamon J, Waki H, Murakami K, Motojima K, Komeda K et al. The mechanisms by which both heterozygous peroxisome proliferatoractivated receptor gamma (PPARgamma) deficiency and PPARgamma agonist improve insulin resistance. J Biol Chem 2001; 276: 41245-41254.

30 Matsushita K, Wu Y, Okamoto Y, Pratt RE, Dzau VJ. Local renin angiotensin expression regulates human mesenchymal stem cell differentiation to adipocytes. Hypertension 2006; 48: 1095-1102.

31 Werner C, Kamani CH, Gensch C, Böhm M, Laufs U. The peroxisome proliferator-activated receptor-gamma agonist pioglitazone increases number and function of endothelial progenitor cells in patients with coronary artery disease and normal glucose tolerance. Diabetes 2007; 56: 2609-2615.

32 Vosough M, Moossavi S, Mardpour S, Akhlaghpoor S, Azimian V, Jarughi N et al. Repeated intraportal injection of mesenchymal stem cells in combination with pioglitazone in patients with compensated cirrhosis: a clinical report of two cases. Arch Iran Med 2016; 19: 131-136.

33 Wang L, Li L, Gao H, Li Y.. Effect of pioglitazone on transdifferentiation of preosteoblasts from rat bone mesenchymal stem cells into adipocytes. J Huazhong Univ Sci Technolog Med Sci 2012; 32: 530-533.

34 Hou J, Wang L, Hou J, Guo T, Xing Y, Zheng S et al. Peroxisome proliferator-activated receptor gamma promotes mesenchymal stem cells to express connexin43 via the inhibition of TGF- $\beta 1 /$ smads signaling in a rat model of myocardial infarction. Stem Cell Rev 2015; 11: 885-899.

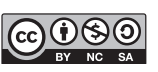

This work is licensed under a Creative Commons Attribution-NonCommercial-ShareAlike 4.0 International License. The images or other third party material in this article are included in the article's Creative Commons license, unless indicated otherwise in the credit line; if the material is not included under the Creative Commons license, users will need to obtain permission from the license holder to reproduce the material. To view a copy of this license, visit http://creativecommons.org/licenses/by-nc-sa/4.0/ 